\title{
Thinning ferroelectric films for high- efficiency photovoltaics based on the Schottky barrier effect
}

\author{
Zhengwei Tan ${ }^{1,2}$, Lanqing Hong ${ }^{3}$, Zhen Fan (1) ${ }^{1,2}$, Junjiang Tian' ${ }^{1}$ Luyong Zhang ${ }^{1}$, Yue Jiang ${ }^{1}$, Zhipeng Hou' \\ Deyang Chen ${ }^{1}$, Minghui Qin', Min Zeng ${ }^{1}$, Jinwei Gao ${ }^{1}$, Xubing Lu' ${ }^{1}$ Guofu Zhou ${ }^{2,4}$, Xingsen Gao ${ }^{1}$ and \\ Jun-Ming Liu (iD ${ }^{1,5}$
}

\begin{abstract}
Achieving high power conversion efficiencies (PCEs) in ferroelectric photovoltaics (PVs) is a longstanding challenge. Although recently ferroelectric thick films, composite films, and bulk crystals have all been demonstrated to exhibit PCEs $>1 \%$, these systems still suffer from severe recombination because of the fundamentally low conductivities of ferroelectrics. Further improvement of PCEs may therefore rely on thickness reduction if the reduced recombination could overcompensate for the loss in light absorption. Here, a PCE of up to 2.49\% (under 365-nm ultraviolet illumination) was demonstrated in a 12-nm $\mathrm{Pb}\left(\mathrm{Zr}_{0.2} \mathrm{Ti}_{0.8}\right) \mathrm{O}_{3}$ (PZT) ultrathin film. The strategy to realize such a high PCE consists of reducing the film thickness to be comparable with the depletion width, which can simultaneously suppress recombination and lower the series resistance. The basis of our strategy lies in the fact that the PV effect originates from the interfacial Schottky barriers, which is revealed by measuring and modeling the thickness-dependent PV characteristics. In addition, the Schottky barrier parameters (particularly the depletion width) are evaluated by investigating the thickness-dependent ferroelectric, dielectric and conduction properties. Our study therefore provides an effective strategy to obtain high-efficiency ferroelectric PVs and demonstrates the great potential of ferroelectrics for use in ultrathin-film PV devices.
\end{abstract}

\section{Introduction}

The ferroelectric photovoltaic (PV) effect has gained widespread attention in the past decade ${ }^{1-5}$ because of its promising applications in solar energy harvesting ${ }^{6-8}$, selfpowered photodetection $^{9,10}$, and information storage ${ }^{11,12}$. Ferroelectric PVs exhibit many appealing features that are unavailable in conventional PVs, such as switchable photoresponse $^{13,14}$, above-bandgap photovoltage ${ }^{15}$, and light polarization-dependent photocurrent ${ }^{16}$. Recently, great advances in boosting the power conversion

\footnotetext{
Correspondence: Zhen Fan (fanzhen@m.scnu.edu.cn)

${ }^{1}$ Institute for Advanced Materials, South China Academy of Advanced

Optoelectronics, South China Normal University, Guangzhou 510006, China ${ }^{2}$ Guangdong Provincial Key Laboratory of Optical Information Materials and Technology, South China Academy of Advanced Optoelectronics, South China Normal University, Guangzhou 510006, China
}

Full list of author information is available at the end of the article. efficiencies (PCEs) of ferroelectric PVs have been achieved, including the attainment of PCEs as high as $8.1 \%$ in $\mathrm{Bi}_{2} \mathrm{FeCrO}_{6}$ multilayered films with narrowed bandgaps $(\sim 1.4 \mathrm{eV})^{6}$ and the demonstration of PCE exceeding the Shockley-Queisser limit in $\mathrm{BaTiO}_{3}$ single crystals $^{17}$. Interestingly, the high PCEs $(>1 \%)$ of ferroelectric PVs were achieved mostly in thick films ${ }^{6,18}$, composite films $^{19-21}$, and bulk crystals ${ }^{17}$, all of which have large thicknesses (several hundred nanometers and above). In these systems, severe recombination may occur because ferroelectrics have fundamentally low carrier mobilities and short lifetimes. Hence, reducing the thickness may further improve the PCEs if the reduced recombination could overcompensate for the loss in light absorption. Therefore, the investigation of the PV

\section{(c) The Author(s) 2019}

(c) (i) Open Access This article is licensed under a Creative Commons Attribution 4.0 International License, which permits use, sharing, adaptation, distribution and reproduction c. in any medium or format, as long as you give appropriate credit to the original author(s) and the source, provide a link to the Creative Commons license, and indicate if changes were made. The images or other third party material in this article are included in the article's Creative Commons license, unless indicated otherwise in a credit line to the material. If material is not included in the article's Creative Commons license and your intended use is not permitted by statutory regulation or exceeds the permitted use, you will need to obtain permission directly from the copyright holder. To view a copy of this license, visit http://creativecommons.org/licenses/by/4.0/. 
performances of ferroelectric ultrathin films (a few tens of nanometers and below) is of interest.

A PCE of up to $\sim 18.7 \%$ has been theoretically predicted to exist in 8-nm La-doped $\mathrm{Pb}(\mathrm{Zr}, \mathrm{Ti}) \mathrm{O}_{3}$ (PZT) ultrathin films ${ }^{22}$. However, experiments have shown that only PCEs much lower than $1 \%$ were obtained in $\mathrm{PZT}^{22}, \mathrm{BiFeO}_{3}$ $(\mathrm{BFO})^{23}$, and $\mathrm{BaTiO}_{3}(\mathrm{BTO})^{24}$ ultrathin films. There is apparently a large gap between the predicted and experimental PCEs, and narrowing this gap forms the motivation of this work.

To realize high PCEs in ferroelectric ultrathin films, it is necessary to understand the PV mechanisms beforehand. Below, we will highlight several widely accepted PV mechanisms discovered in ferroelectric films:

i. Bulk photovoltaic effect (BPVE). The BPVE manifests itself as a steady photocurrent generated in the homogeneous bulk region, which originates from the lattice noncentrosymmetry-induced asymmetric momentum distribution of photoexcited carriers $^{24-27}$.

ii. Depolarization field $\left(E_{\mathrm{dp}}\right)$ effect. The $E_{\mathrm{dp}}$, which arises from the incomplete screening of polarization and exists across the whole film, can drive the separation of photoexcited carriers ${ }^{28,29}$.

iii. Schottky barrier effect. A Schottky barrier is generally formed at the ferroelectric/electrode interface, and the barrier height and width can be modulated by polarization. The built-in field $\left(E_{\mathrm{bi}}\right)$ in the depletion region provides the driving force for the PV effect ${ }^{30-35}$, similar to that in typical junction-based solar cells.

iv. Domain wall effect. The potential step developed at an individual ferroelectric domain wall can separate the photoexcited carriers, and the photovoltages of periodically ordered domain walls are additive ${ }^{15,36}$.

Adding to the complexity of PV effects in ferroelectric films, more than one of the above mechanisms may coexist in a single ferroelectric film ${ }^{37,38}$. Nevertheless, only one mechanism is suitable for realizing high PCEs in ferroelectric ultrathin films, which can be analyzed as follows. Because the BPVE and $E_{\mathrm{dp}}$ are bulk effects, the open-circuit voltage $\left(V_{\mathrm{OC}}\right)$ they generate would increase with thickness ${ }^{25,29}$. The $V_{\mathrm{OC}}$ generated by the domain wall effect would also scale with thickness if the density of periodically ordered domain walls is uniform along the thickness direction. It therefore seems that the BPVE, $E_{\mathrm{dp}}$, and domain wall effects are all unable to retain a sizable $V_{\mathrm{OC}}$, a prerequisite for a high PCE, in ultrathin films.

Our focus thus turns to the Schottky barrier effect, which requires only a very small region (i.e., the depletion region) to induce the PV effect. Indeed, the photoexcited carriers are most efficiently separated in the depletion region where $E_{\mathrm{bi}}$ exists. Outside the depletion region, although light absorption and carrier generation still occur, carrier recombination may dominate because of the very small diffusion lengths of ferroelectrics (typically a few nanometers $)^{36}$. Therefore, for the Schottky barrierdriven PV effect, reducing the film thickness down to a value comparable with the depletion width may lead to high PCEs, i.e., ferroelectric ultrathin films may have higher PCEs than their thicker counterparts.

Herein, to implement the above strategy, we chose to investigate $\mathrm{Pb}\left(\mathrm{Zr}_{0.2} \mathrm{Ti}_{0.8}\right) \mathrm{O}_{3}$ ( $\mathrm{PZT}$ 20/80) epitaxial thin films. PZT $20 / 80$ is a prototype ferroelectric exhibiting large polarization $\left(>60 \mu \mathrm{C} / \mathrm{cm}^{2}\right), n$-type semiconducting characteristics $^{39}$, and strong photoresponse in the ultraviolet (UV) wavelength region ${ }^{31}$. Through a combination of polarization-voltage $(P-V)$, capacitance-voltage $(C-V)$, and dark current-voltage $(I-V)$ measurements on $\mathrm{Pt} / \mathrm{PZT} / \mathrm{SrRuO}_{3}$ (SRO) structures with different PZT thicknesses, we revealed that the Schottky barriers play significant roles in polarization switching, dielectric response, and conduction; furthermore, we extracted the Schottky barrier parameters. Then, we observed switchable PV behaviors, where $V_{\mathrm{OC}}$ depends weakly on thickness and photocurrent decreases with thickness, both of which can be well described by a Schottky barrier model. Based on the experimental and modeling results, we designed and fabricated a 12-nm PZT film whose thickness was close to the depletion width and demonstrated a PCE of $2.49 \%$, which was extremely high for such thin ferroelectrics.

\section{Materials and methods}

The PZT 20/80 films with thicknesses ranging from 4 to $300 \mathrm{~nm}$ were grown on SRO-buffered $\mathrm{SrTiO}_{3}$ (STO) (001) substrates using pulsed laser deposition (PLD) (KrF excimer laser, $\lambda=248 \mathrm{~nm})$. The SRO layers $(\sim 50 \mathrm{~nm})$ were first deposited on the STO substrates at a temperature of $680^{\circ} \mathrm{C}$ and an oxygen pressure of $15 \mathrm{~Pa}$. Subsequently, the PZT layers were grown on top of the SRO layers at a lower temperature of $600^{\circ} \mathrm{C}$ and the same oxygen pressure of $15 \mathrm{~Pa}$. After growth, the films were cooled to room temperature at $10^{\circ} \mathrm{C} / \mathrm{min}$ in an oxygen atmosphere of $1 \mathrm{~atm}$. The Pt electrodes (diameter: $\sim 200$ $\mu \mathrm{m}$; thickness: $\sim 10 \mathrm{~nm}$ ) were ex situ deposited on the PZT films by PLD through a shadow mask at room temperature and under vacuum $\left(\sim 10^{-4} \mathrm{~Pa}\right)$. To reduce leakage currents in the ultrathin PZT films, Pt top electrodes of only $\sim 5 \mu \mathrm{m}$ in diameter were used and deposited by PLD through a mask patterned by electron beam lithography (EBL). We used Pt rather than SRO as top electrodes in this study to achieve both good conductivity and transmittance.

The crystal structures and epitaxial qualities were examined using X-ray diffraction (XRD) and reciprocalspace mapping (RSM) (X'Pert PRO, PANalytical, The 
Netherlands). Piezoresponse force microscopy (PFM), conductive atomic force microscopy (C-AFM), and scanning Kelvin probe microscopy (SKPM) characterizations were performed with a commercial atomic force microscope (Cypher, Asylum Research, UK) and Pt-coated silicon tips (EFM Arrow, Nanoworld, Switzerland). The $P-V$ hysteresis loops, $C-V$, and $I-V$ characteristics were measured using a ferroelectric workstation (Precision Multiferroic, Radiant, USA), an inductance-capacitanceresistance (LCR) meter (E4980A, Agilent, USA), and a source meter (6430, Keithley, USA), respectively. Voltages were applied to the top electrode while the bottom electrode was grounded. The photovoltaic properties were measured using a setup comprising the source meter and a UV light-emitting diode (LED) (light wavelength: $365 \mathrm{~nm}$ ). The UV light was unpolarized, and it was illuminated along the normal direction of the sample surface.

\section{Results and discussion Crystalline phases}

Figure 1a shows the XRD $\theta-2 \theta$ diffraction patterns of the PZT/SRO bilayers grown on the STO substrates. The diffraction peaks all arise from the $(00 l)$ reflections of PZT, SRO, and STO, indicating epitaxial growth and good phase purity. Furthermore, the PZT (002) peak splits into two, where one peak is located at $43.59^{\circ}$ while the other one shifts from $42.35^{\circ}$ to $42.63^{\circ}$ as the thickness increases from 120 to $300 \mathrm{~nm}$. The peak at $43.59^{\circ}$ corresponds to an out-of-plane lattice constant of $c_{1}=4.143 \AA$, which is quite close to that of the bulk PZT $\left(c_{\text {bulk }}=4.132 \AA\right)^{40}$. However, the peaks in the range of $42.35^{\circ}$ to $42.63^{\circ}$ correspond to larger lattice constants: $c_{2}=4.232-4.259 \AA$. These results suggest that our PZT films may contain two tetragonal phases: a bulk-like $\mathrm{T}_{1}$ phase that exists near the top of the film and is almost strainfree and a strained $T_{2}$ phase that exists near the bottom of the film and undergoes lattice relaxation as the film becomes thicker. To obtain the in-plane structural information, the (103) RSM was performed for the 120-nm PZT film (see Fig. 1b). There are two clear diffraction spots of the PZT (103) reflection, where one spot has almost the same $\mathrm{H}$ coordinate as that of STO, while the other has a smaller $\mathrm{H}$-coordinate. The RSM results also suggest the coexistence of two tetragonal phases: one is highly strained with an in-plane lattice constant of $a_{2}=3.912 \AA$ (i.e., the $\mathrm{T}_{2}$ phase), while the other is strain-relaxed with $a_{1}=$ $3.948 \AA$ (i.e., the $T_{1}$ phase). Our observation that two tetragonal phases coexist in the PZT 20/80 epitaxial films grown on the SRO-buffered STO substrates is consistent with previous reports ${ }^{40,41}$. Notably, the strain-induced phase inhomogeneity in the PZT films will not affect the question of interest in this study (i.e., thickness reduction for potentially high PCEs) because the strained and strain-relaxed phases have similar electrical and optical properties, e.g., polarization ${ }^{42}$ (to be shown later) and bandgap ${ }^{43}$.

\section{Microscopic characterizations of ferroelectricity}

Figure $2 \mathrm{a}-\mathrm{d}$ shows the surface topography, out-of-plane PFM amplitude and phase images, and local PFM hysteresis loops of a representative 120-nm PZT film. The surface of the film is quite flat, with a small roughness of only $370 \mathrm{pm}$. Upon further examination of the topography and amplitude images (Fig. 2a, b), one can see a grid of needle-like $a$-domains embedded into the matrix of $c$ domains. This type of domain structure is a typical feature of PZT 20/80 epitaxial thin films ${ }^{41,44,45}$. In addition, two square regions of $5 \times 5 \mu \mathrm{m}^{2}$ (outer) and $3 \times 3 \mu \mathrm{m}^{2}$ (inner)
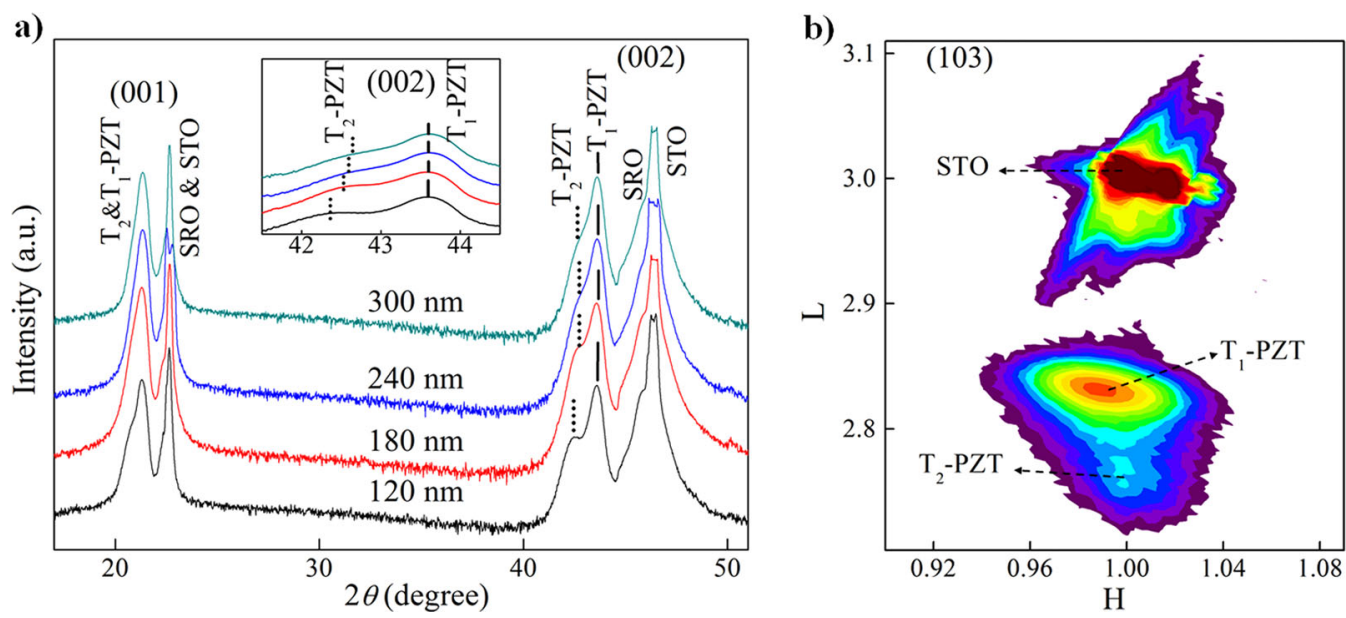

Fig. 1 XRD and RSM results. a XRD $\theta-2 \theta$ scans of the PZT films with thicknesses of 120, 180, 240, and 300 nm grown on the SRO-buffered STO substrates. The inset shows the magnified (002) diffraction peaks of PZT. b RSM around the (103) reflection for the 120-nm PZT film 

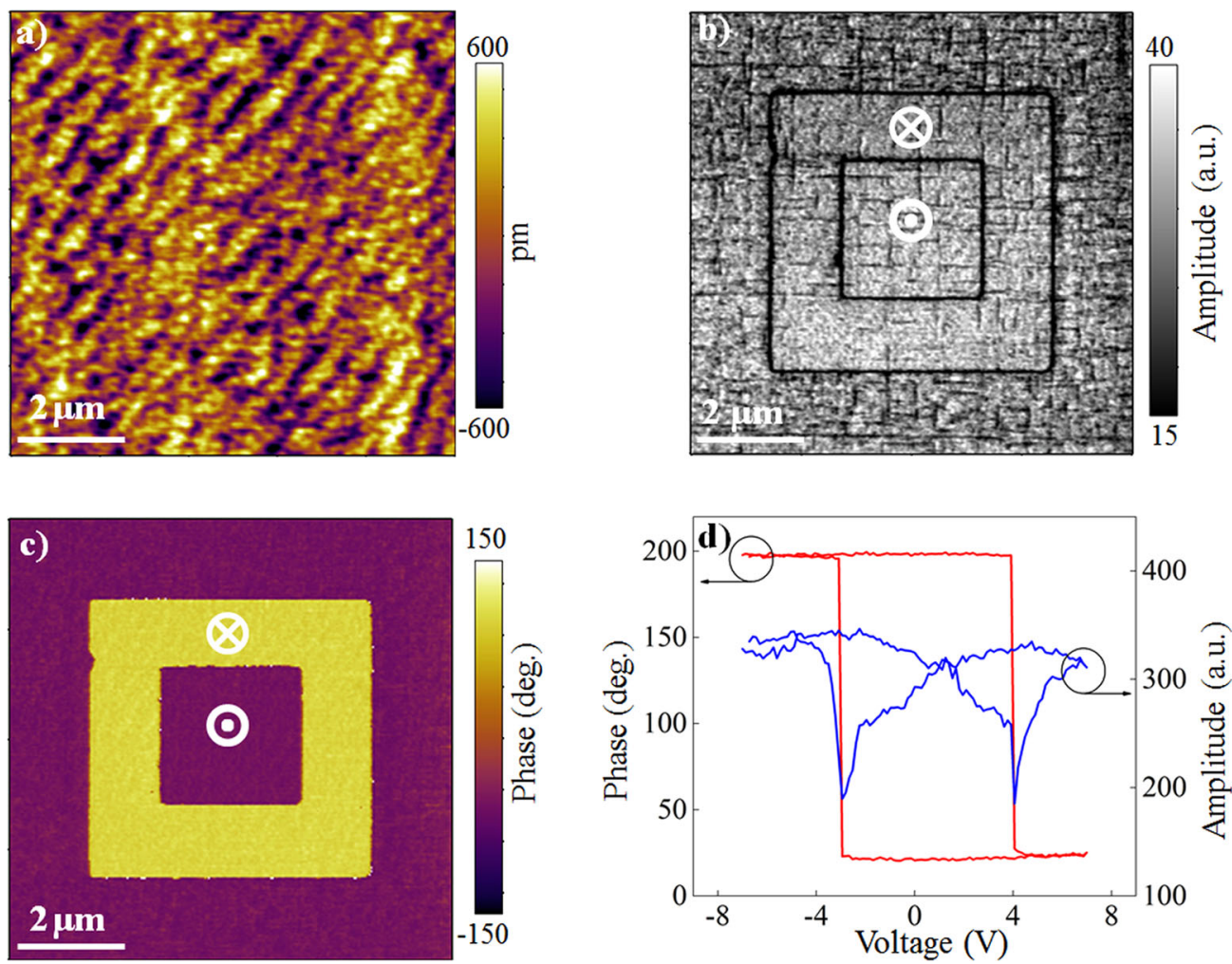

Fig. 2 Topography and nanoscale ferroelectricity. a Topography, b out-of-plane PFM amplitude and $\mathbf{c}$ phase images, and $\mathbf{d}$ local PFM hysteresis loops of the 120-nm PZT film

were electrically written with $+6 \mathrm{~V}$ and $-6 \mathrm{~V}$, respectively. The resultant PFM phase image (Fig. 2c) shows that the $-6 \mathrm{~V}$-written region and the as-grown region have the same phase contrast, but there is a phase difference of $180^{\circ}$ between the $+6 \mathrm{~V}$-written and $-6 \mathrm{~V}$-written regions. Therefore, the as-grown PZT film is self-polarized, in which the polarization of the dominant $c$-domains is upward and can be switched by writing with $+6 \mathrm{~V}$. In addition, the out-of-plane PFM amplitude and phase loops (Fig. 2d) exhibit a butterfly-like shape and a sharp $180^{\circ}$ switching, respectively, confirming the ferroelectricity of the PZT film.

\section{$P-V$ hysteresis loops}

To further characterize ferroelectricity, macroscopic $P-V$ hysteresis loops were measured for the $\mathrm{Pt} / \mathrm{PZT} / \mathrm{SRO}$ structures with PZT thicknesses of 120, 180, 240, and 300 $\mathrm{nm}$. As shown in Fig. 3a, all the films exhibit square $P-V$ loops with large remnant polarization $\left(P_{\mathrm{r}}\right)$ values up to $\sim 80 \mu \mathrm{C} / \mathrm{cm}^{2}$. Previously, Pintilie et al. ${ }^{33,39,46}$ obtained record-high $P_{\mathrm{r}}$ values of $\sim 100 \mu \mathrm{C} / \mathrm{cm}^{2}$ regardless of the film thickness in their defect-free PZT 20/80 epitaxial thin films. Their results are largely consistent with ours in spite of the lower $P_{\mathrm{r}}$ values in our PZT films, which may be ascribed to slightly lower crystallinity ${ }^{46}$. Recall that as the film thickness increases, the strain relaxation induces a phase transition in our PZT films. Nevertheless, $P_{\mathrm{r}}$ is found to be almost independent of thickness, which can be explained by the insensitivity of the $\mathrm{Pb}$ displacement to strain $^{42}$.

Another interesting observation is that the coercive voltage $\left(V_{\mathrm{c}}\right)$ varies only slightly as the thickness increases (Fig. 3a, b), similar to other reports ${ }^{39,41}$. The weak dependence of $V_{\mathrm{c}}$ on thickness may be associated with interface-controlled domain nucleation. More specifically, the electrical field required for domain nucleation is generally much higher than that required for domain growth in ferroelectric films ${ }^{47,48}$; hence, domain nucleation is the critical step of polarization switching. On the other hand, for a metal-ferroelectric-metal (MFM) structure with two interfacial Schottky barriers, the voltage drop occurs mostly on the reverse-biased barrier rather than the bulk region. That is, the highest electric field appears at the interfacial Schottky barrier, and this field triggers domain nucleation. Therefore, $V_{\mathrm{c}}$ depends weakly on the dimension of the bulk region (i.e., the film thickness). In addition, the offset of $V_{\mathrm{c}}$ along the voltage axis (Fig. 3a) is related to the asymmetric $E_{\mathrm{bi}}$ in the top and bottom Schottky barriers (see detailed discussion in Sec. I in Supplementary Information). 


\section{C-V characteristics}

The role of interfacial Schottky barriers was also probed by the $C-V$ measurements. Figure 4 a summarizes the thickness-dependent $C-V$ characteristics. All the PZT
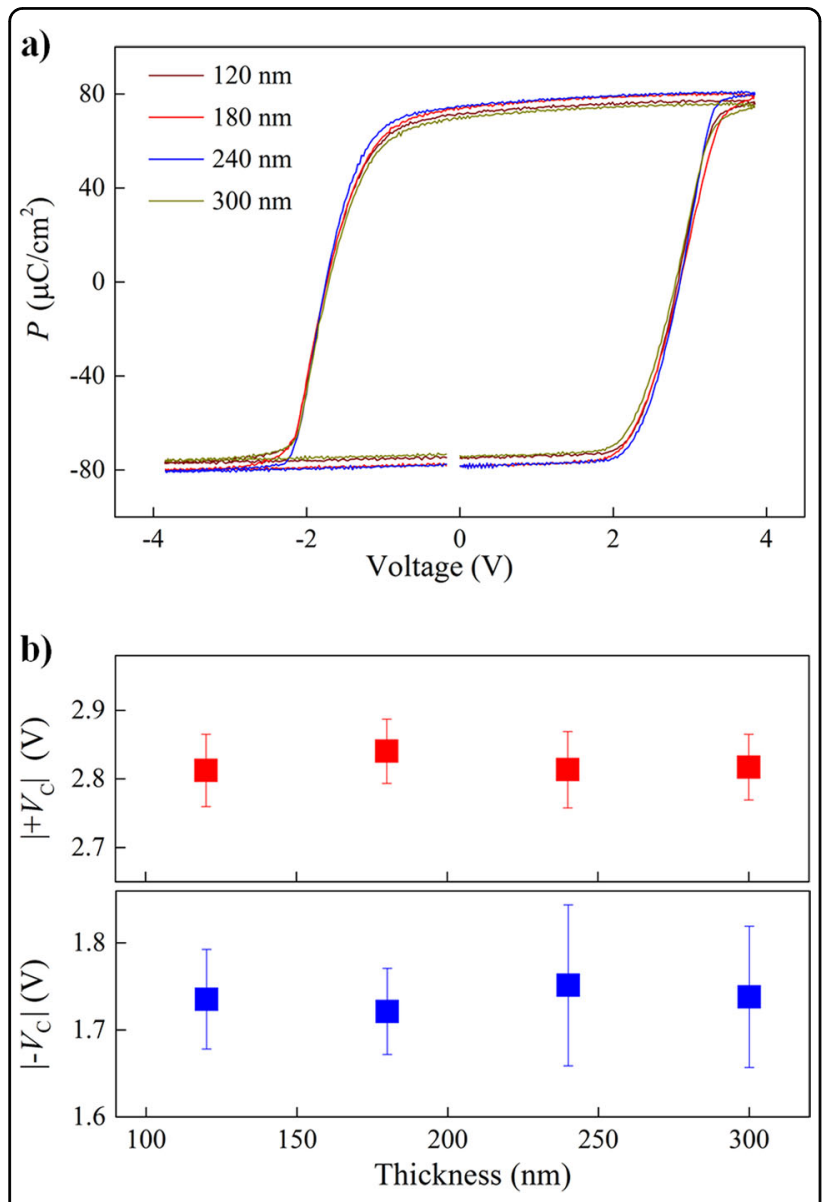

Fig. 3 Macroscopic ferroelectric properties. a $P-V$ hysteresis loops of Pt/PZT/SRO capacitors with different PZT thicknesses (frequency: 3.3 $\mathrm{kHz})$. $\mathbf{b}$ Positive and negative coercive voltages as a function of thickness films clearly exhibit butterfly-like $C-V$ curves with two capacitance peaks, indicating polarization switching. Notably, the $V_{c}$ values obtained from the $C-V$ characteristics $\left(+V_{\mathrm{c}}=1.4 \mathrm{~V}\right.$ and $\left.-V_{\mathrm{c}}=-1.1 \mathrm{~V}\right)$ are smaller than those obtained from the $P-V$ loops (Fig. $3 \mathrm{~b}$ ), which is probably due to the different polarization switching dynamics in the different voltage sweeping modes (note: the DC and high-frequency AC voltages were applied in the $C-V$ and $P-V$ measurements, respectively). Nevertheless, the $C-V$ characteristics show that the $V_{\mathrm{c}}$ values are largely independent of thickness and exhibit positive offsets, which is consistent with the results revealed by the $P-V$ loops.

Figure 4a also shows that the capacitance at zero voltage decreases as the film thickness increases (an average capacitance is taken if there are two intercepts on the capacitance axis). The reciprocal of the zero-voltage capacitance as a function of thickness $(1 / C-d)$ is plotted in Fig. 4b, which shows good linearity. By linearly extrapolating the $1 / C-d$ curve to $d=0$, a finite value of $1 / C$ $\left(9.7 \times 10^{8} \mathrm{~F}^{-1}\right)$ is obtained. This finding indicates that there are dielectric responses from the interfacial Schottky barriers $^{30}$, which is explained in detail in Sec. II in Supplementary Information.

In addition, Fig. 4a shows that capacitance continues to decrease with increasing |voltage| in the voltage regions where the polarization switching is completed (e.g., $>2.5 \mathrm{~V}$ and $<-1.5 \mathrm{~V}$ ). This phenomenon may be attributed to the increase in the Schottky barrier width as the reverse bias increases ${ }^{49}$, further confirming the formation of Schottky barriers at both the top and bottom interfaces. Using the plots of $1 / C^{2}$ versus $V$ for a representative $120-\mathrm{nm}$ film, as shown in Fig. 4c, one can estimate the free carrier concentration $\left(n_{\text {free }}\right)$, an important Schottky barrier parameter (see Sec. II in Supplementary Information for details). The average value of $n_{\text {free }}$ is $6.7 \times 10^{24} \mathrm{~m}^{-3}$, which is close to the values reported for the PZT 20/80 epitaxial thin films ${ }^{41,50}$. Notably, we determined that our PZT is an
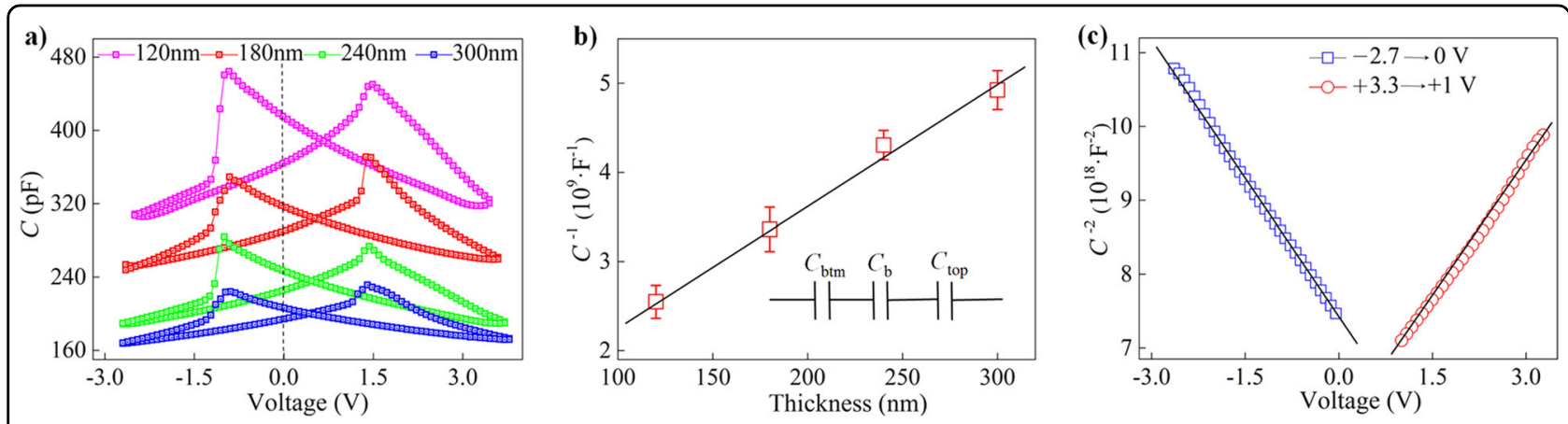

Fig. 4 Dielectric properties. a Thickness-dependent C-V characteristics of the PZT films. $\mathbf{b}$ Inverse zero-voltage capacitance as a function of thickness. $\mathbf{c} 1 / C^{2}-\mathrm{V}$ relationships of a 120-nm film in the voltage regions of $-2.7 \mathrm{~V} \rightarrow 0 \mathrm{~V}$ and $+3.3 \mathrm{~V} \rightarrow+1 \mathrm{~V}$, where the capacitance is least influenced by the polarization switching 
$n$-type semiconductor (oxygen vacancies may act as donors $)^{39}$; therefore, the free carriers are indeed electrons (see Fig. S1 in Supplementary Information for evidence).

\section{Dark $I-V$ characteristics}

To gain more information about the Schottky barriers, we then investigated the conduction behavior. The dark $I-V$ characteristics were measured for the Pt/PZT/SRO structures with PZT thicknesses of 120, 180, 240, and 300 $\mathrm{nm}$. To avoid the polarization switching-induced negative differential resistance effect ${ }^{51}$, polarization was first switched to the $P_{\text {down }}\left(P_{\text {up }}\right)$ direction by a $+4 \mathrm{~V}(-4 \mathrm{~V})$ pulse, and then a DC voltage sweep of $0 \rightarrow+4 \mathrm{~V}(0 \rightarrow-3 \mathrm{~V})$ was applied. The resultant $I-V$ characteristics are displayed in Fig. 5a. The $I-V$ characteristics of the PZT films with different thicknesses are surprisingly similar and are all

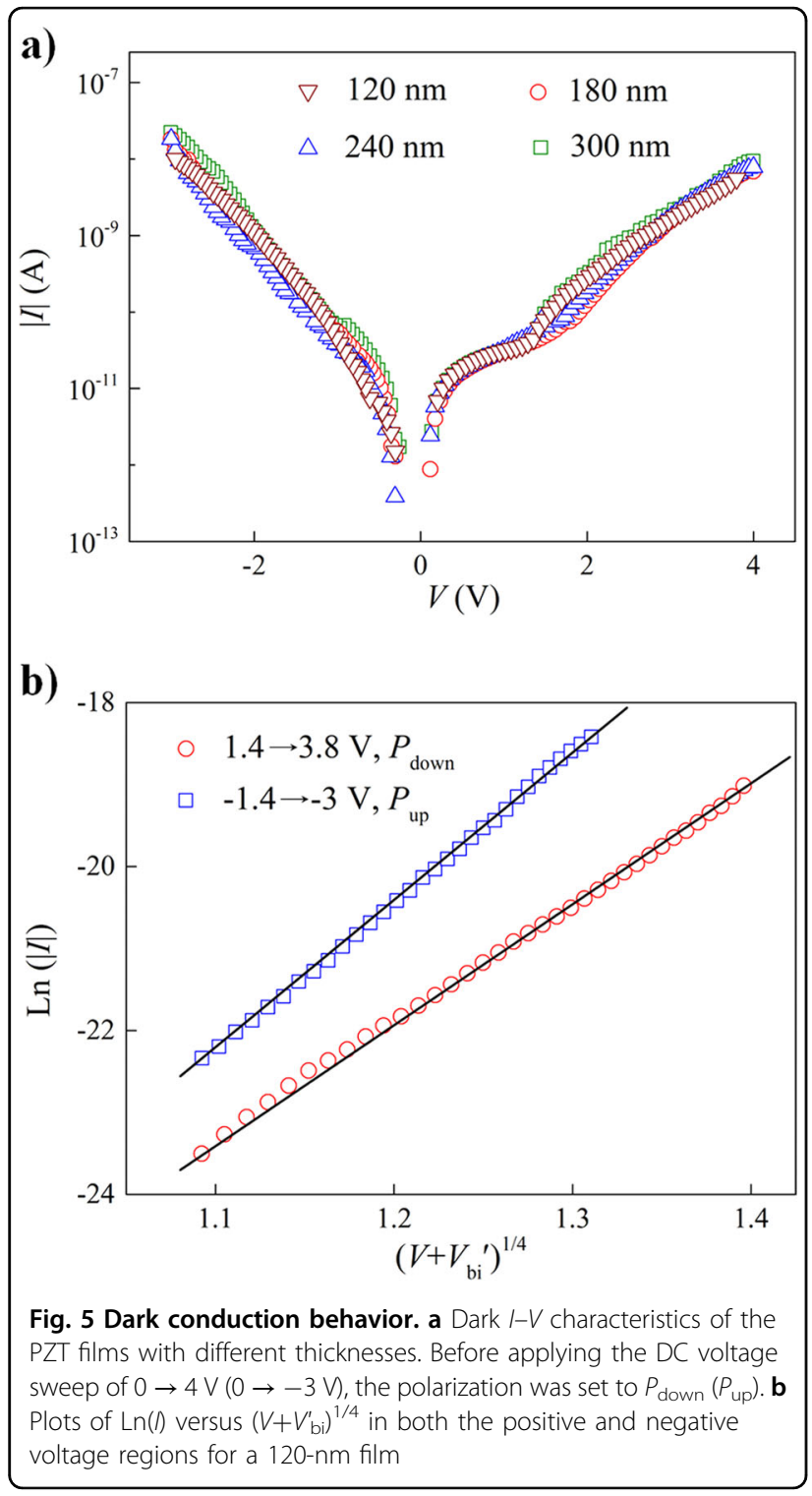

asymmetric. This observation is consistent with that reported by Pintilie et al. ${ }^{39}$, and strongly indicates that the conduction mechanism is interface-limited rather than bulk-limited.

Furthermore, some typical interface-limited conduction models, including the Schottky emission and Fowler-Nordheim tunneling, were used to fit the experimental $I-V$ data. The Fowler-Nordheim tunneling was excluded as the conduction mechanism, because the fitting based on this model yielded unreasonably small barrier heights. However, the Schottky emission model can fit the $I-V$ characteristics well, as indicated by the good linearities of the $\operatorname{Ln}(I)-\left(V+V_{\mathrm{bi}}^{\prime}\right)^{1 / 4}$ curves in both positive and negative voltage regions for a representative 120-nm film (see Fig. 5b). Here, $V_{\mathrm{bi}}^{\prime}$ is the apparent builtin voltage, and the detailed fitting procedures are presented in Sec. III in Supplementary Information. From the slopes of the $\operatorname{Ln}(I)-\left(V+V_{\mathrm{bi}}^{\prime}\right)^{1 / 4}$ curves, the values of the effective charge concentration $\left(N_{\text {eff }}\right)$ are calculated to be $1.5 \times 10^{27}$ and $4.6 \times 10^{27} \mathrm{~m}^{-3}$ for the PZT/SRO and Pt/ PZT barriers, respectively. These values are on the same order of magnitude as those reported for the PZT films ${ }^{50,52}$, and the difference in $N_{\text {eff }}$ between the top and bottom barriers implies a nonuniform distribution of charged defects (e.g., oxygen vacancies). In addition, the barrier heights resulting from the band alignment without considering the polarization modulation $\left(\Phi^{0}{ }_{\mathrm{B}}\right)$ can be extracted from the intercepts of the $\operatorname{Ln}(I)-\left(V+V^{\prime}{ }_{\mathrm{bi}}\right)^{1 / 4}$ curves to be 1.24 and $1.34 \mathrm{eV}$ for the PZT/SRO and Pt/ PZT barriers, respectively. These values are not equal to those obtained from the Schottky-Mott rule, which might be due to the Fermi-level pinning effect. (Note: the work functions of Pt and SRO are $\sim 5.3$ and $\sim 5.2 \mathrm{eV}$, respectively. In addition, the electron affinity of PZT is $\sim 3.5$ $\mathrm{eV}^{53}$.)

Through the fittings, polarization-induced band modulation $^{32,35,54-56}$ can also be revealed. For the PZT/SRO (Pt/PZT) barrier in the $P_{\text {down }}\left(P_{\text {up }}\right)$ state, the barrier is flattened with $V_{\mathrm{bi}}^{\prime}=0$. In contrast, for the PZT/SRO (Pt/ PZT) barrier in the $P_{\text {up }}\left(P_{\text {down }}\right)$ state, the $V_{\text {bi }}^{\prime}$ is increased to $2.28 \mathrm{~V}(2.48 \mathrm{~V})$. Then, the depletion widths $(W)$ can be calculated by

$$
W=\sqrt{\frac{2 \varepsilon_{0} \varepsilon_{s t}\left(V+V_{b i}^{\prime}\right)}{q N_{e f f}}},
$$

where $q$ is the electron charge, $\varepsilon_{0}$ is the vacuum permittivity, and $\varepsilon_{\mathrm{st}}$ is the static dielectric constant of the Pt/ $\mathrm{PZT} / \mathrm{SRO}$ structure. At $V=0$, the $W$ value of the PZT/ SRO $(\mathrm{Pt} / \mathrm{PZT})$ barrier in the $P_{\text {up }}\left(P_{\text {down }}\right)$ state is estimated to be $5.4 \mathrm{~nm}(3.2 \mathrm{~nm})$.

With these results, one can construct energy band diagrams for the $\mathrm{Pt} / \mathrm{PZT} / \mathrm{SRO}$ structure, as shown in Fig. 6. In the absence of polarization, two back-to-back 

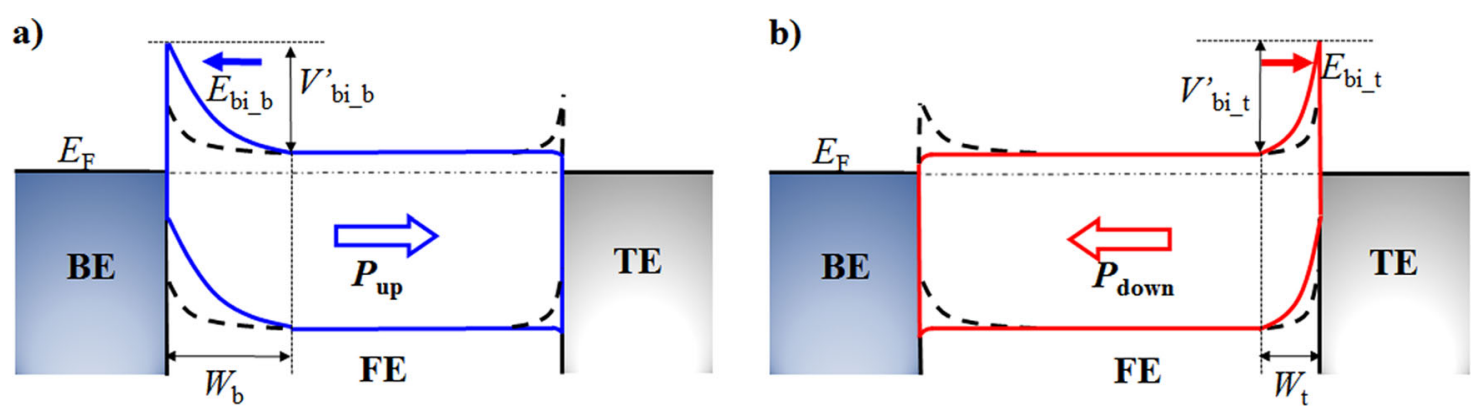

Fig. 6 Energy band modulation by polarization. Schematic energy band diagrams of the MFM structure in the $\mathbf{a} P_{\text {up }}$ and $\mathbf{b} P_{\text {down }}$ states. The abbreviations $B E$, FE, and TE indicate the bottom electrode (SRO), ferroelectric film (PZT), and top electrode (Pt), respectively. The depletion widths of $W_{\mathrm{b}}$ and $W_{\mathrm{t}}$ are calculated to be 5.4 and $3.2 \mathrm{~nm}$, respectively. The bold dashed lines indicate the energy band in the absence of polarization

Schottky barriers with moderate heights are formed at the $\mathrm{PZT} / \mathrm{SRO}$ and Pt/PZT interfaces (bold dashed lines in Fig. 6a, b). In the $P_{\text {up }}$ state, the built-in voltage of the PZT/ SRO barrier is enhanced $\left(V_{\mathrm{bi} b}^{\prime}=2.28 \mathrm{~V}\right)$, and the depletion region becomes wider $\left(W_{\mathrm{b}}=5.4 \mathrm{~nm}\right)$, while the $\mathrm{Pt} /$ PZT barrier is flattened (Fig. 6a). In the $P_{\text {down }}$ state, however, the reverse scenario occurs, i.e., the Pt/PZT barrier is enhanced $\left(V_{\text {bi } \mathrm{t}}^{\prime}=2.48 \mathrm{~V}\right.$ and $\left.W_{\mathrm{t}}=3.2 \mathrm{~nm}\right)$, while the PZT/SRO barrier is flattened (Fig. 6b). The above Schottky barrier parameters (particularly the depletion width) provide guidance for optimizing thickness to achieve high PV performance. Before optimizing, the Schottky barrier effect should be confirmed as the major PV mechanism. The thickness-dependent PV characteristics were therefore investigated, and the results are shown below.

\section{Thickness-dependent PV characteristics}

Figure $7 \mathrm{a}, \mathrm{b}$ shows the $I-V$ characteristics of the PZT films $(120,180,240$, and $300 \mathrm{~nm})$ in the $P_{\text {up }}$ and $P_{\text {down }}$ states under 365-nm UV illumination (intensity: $170 \mathrm{~mW} / \mathrm{cm}^{2}$ ). Obviously, all the PZT films exhibit switchable PV behaviors, and the signs of open-circuit voltage $\left(V_{\mathrm{OC}}\right)$ and shortcircuit current $\left(I_{\mathrm{SC}}\right)$ can be fully reversed. Table 1 summarizes the thickness-dependent $V_{\mathrm{OC}}, I_{\mathrm{SC}}, J_{\mathrm{SC}}$ (i.e., $I_{\mathrm{SC}} / A$ ), fill factor (FF), and PCE values. $V_{\mathrm{OC}}$ remains almost unchanged as thickness increases, i.e., $\sim-0.9 \mathrm{~V}$ in the $P_{\text {up }}$ state and $\sim 0.36 \mathrm{~V}$ in the $P_{\text {down }}$ state. $J_{\mathrm{SC}}$ decreases monotonically with increasing thickness in both the $P_{\text {up }}$ and $P_{\text {down }}$ states. The values of FF and PCE also show decreasing trends with thickness. The PCE value obtained in the $P_{\text {up }}$ state of the $120-\mathrm{nm}$ film reaches $0.51 \%$, which is the highest among our samples. Figure 7c shows the photocurrents measured by applying cyclic ON/OFF illuminations, demonstrating that the photoresponses are fast, stable, and reproducible.

We then analyzed the PV mechanism. Figure $7 \mathrm{a}, \mathrm{b}$ shows that most of the $I-V$ characteristics are curved rather than linear, suggesting that the Schottky barriers may play a role in the PV effect. Moreover, the weak dependence of $V_{\mathrm{OC}}$ on thickness excludes BPVE and $E_{\mathrm{dp}}$ as the major PV mechanisms. In addition, the number of domain walls in the poled states is very small (see Fig. 2 b, c), which excludes the domain wall effect. Therefore, we deduced that the Schottky barrier effect is the dominant PV mechanism.

More specifically, the PZT/SRO and Pt/PZT Schottky barriers mainly contribute to the PV effects in the $P_{\text {up }}$ and $P_{\text {down }}$ states, respectively (see Fig. 6 and Fig. S1 in Supplementary Information). The $E_{\mathrm{bi}}$ in the $\mathrm{PZT} / \mathrm{SRO}(\mathrm{Pt} /$ PZT) barrier is downward (upward), thus resulting in a negative (positive) $V_{\mathrm{OC}}$. This observation is consistent with the observed negative (positive) $V_{\mathrm{OC}}$ in the $P_{\text {up }}$ $\left(P_{\text {down }}\right)$ state (see Fig. 7a, b). In addition, the asymmetric $\mathrm{PV}$ behaviors can be explained by the asymmetry of the PZT/SRO and Pt/PZT barriers.

Therefore, the PV effects in both the $P_{\text {up }}$ and $P_{\text {down }}$ states may be described by a single-barrier solar cell model. This model's equivalent circuit is schematically drawn in Fig. 7d, which consists of a Schottky diode $(D)$, a constant current source $\left(I_{\mathrm{ph}}\right.$, i.e., the photocurrent, which should be distinguished from $\left.I_{\mathrm{SC}}\right)$, a series resistance $\left(R_{\mathrm{S}}\right)$ and a shunt resistance $\left(R_{\mathrm{sh}}\right)$. The $I-V$ relationship is given by

$$
I=I_{0}\left[\exp \left(q \frac{V-R_{s} I}{n k T}\right)-1\right]+\frac{V-R_{s} I}{R_{s h}}-I_{p h}
$$

where $I_{0}$ is the reverse saturation current, $n$ is the ideality factor, $T$ is temperature, and $\mathrm{k}$ is the Boltzmann constant. Equation (2) was used to fit the experimental illuminated $I-V$ characteristics by employing the Lambert $\mathrm{W}$ function $^{57-59}$. Figure $7 \mathrm{a}, \mathrm{b}$ shows that the calculated $I-V$ curves fit the experimental data fairly well, demonstrating the validity of the Schottky barrier model.

Using the above fittings, the parameters $I_{\mathrm{ph}}, R_{\mathrm{s}}$, and $R_{\mathrm{sh}}$ can also be extracted, which are summarized in Table 1 . In both the $P_{\text {up }}$ and $P_{\text {down }}$ states, $I_{\mathrm{ph}}$ decreases while $R_{\mathrm{s}}$ and $R_{\mathrm{sh}}$ increase as the film thickness increases. The 


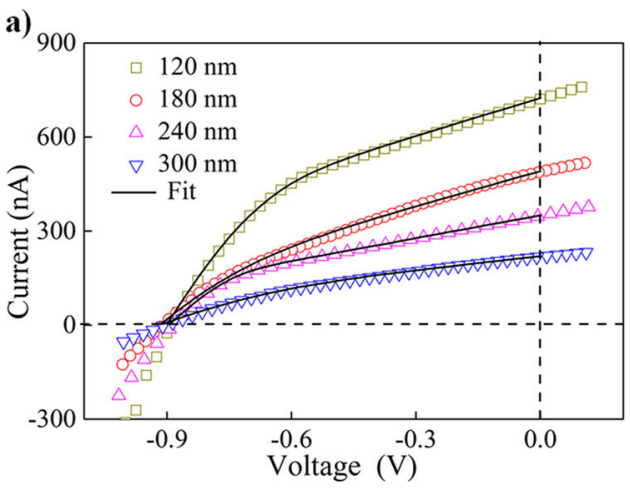

c)

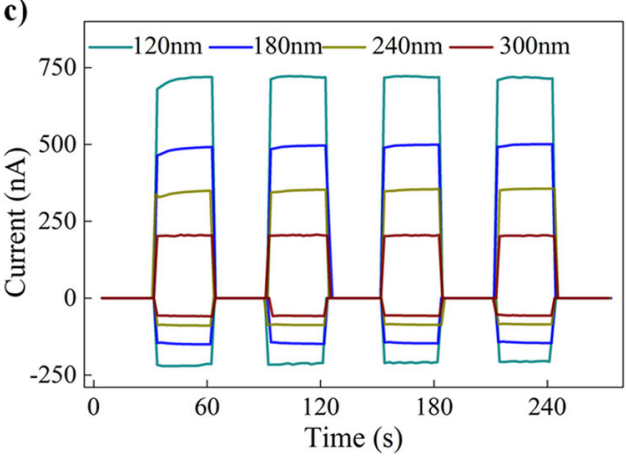

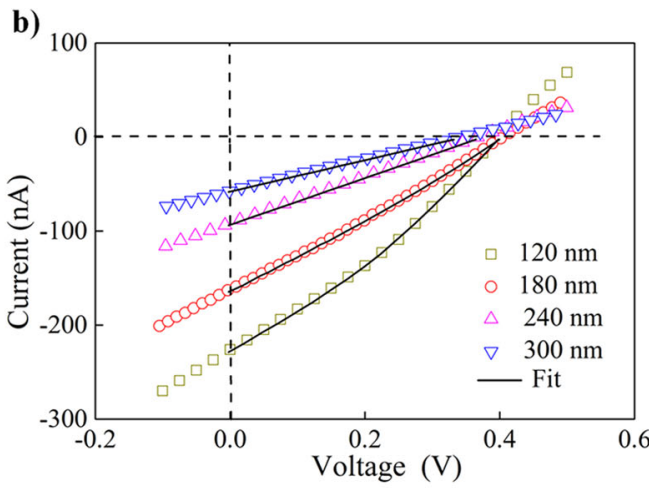

d)

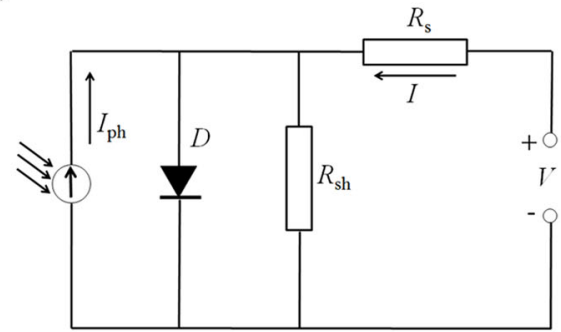

Fig. 7 Photovoltaic characteristics. Illuminated $I-V$ characteristics of the PZT films $(120,180,240$, and $300 \mathrm{~nm})$ in the a $P_{\text {up }}$ and $\mathbf{b} P_{\text {down }}$ states. c Time-resolved photocurrent responses of those PZT films under cyclic ON/OFF illuminations. $\mathbf{d}$ Schematic diagram of an equivalent circuit of the single-barrier solar cell model

Table 1 Thickness-dependent PV properties and fitting parameters of the PZT films

\begin{tabular}{|c|c|c|c|c|c|c|c|c|c|}
\hline Thickness (nm) & $P$ state & $V_{\text {OC }}(\mathrm{V})$ & $I_{\mathrm{SC}}(\mathrm{nA})$ & $J_{\mathrm{SC}}\left(\mathrm{mA} / \mathrm{cm}^{2}\right)$ & FF (\%) & PCE (\%) & $R_{s}(k \Omega)$ & $\mathrm{R}_{\mathrm{sh}}(\mathrm{k} \Omega)$ & $I_{\mathrm{ph}}(\mathrm{nA})$ \\
\hline 120 & $P_{\text {up }}$ & -0.9 & 717 & 2.28 & 41.9 & 0.51 & 343 & 2147 & 836 \\
\hline 180 & & -0.91 & 486 & 1.55 & 33.8 & 0.28 & 469 & 2245 & 590 \\
\hline 240 & & -0.89 & 346 & 1.10 & 39.5 & 0.23 & 627 & 3498 & 409 \\
\hline 300 & & -0.91 & 216 & 0.69 & 35.7 & 0.13 & 1271 & 5534 & 267 \\
\hline 120 & $P_{\text {down }}$ & 0.4 & -225 & -0.72 & 30.7 & 0.051 & 1102 & 1365 & 409 \\
\hline 180 & & 0.39 & -160 & -0.51 & 28.2 & 0.033 & 1180 & 1611 & 282 \\
\hline 240 & & 0.36 & -90 & -0.29 & 26.1 & 0.016 & 2453 & 1644 & 231 \\
\hline 300 & & 0.32 & -55 & -0.18 & 25.6 & 0.009 & 4243 & 1828 & 188 \\
\hline
\end{tabular}

decrease in $I_{\mathrm{ph}}$ with thickness may be due to the enhanced recombination in the thicker films. As the thickness increases, the bulk region becomes wider to allow more occurrences of recombination. We also inferred from the decrease in $I_{\mathrm{ph}}$ that the enhancement in recombination dominates over the gain in light absorption as the thickness increases. On the other hand, the increases in $R_{\mathrm{s}}$ and $R_{\text {sh }}$ may be caused by the larger bulk resistances and fewer shunt paths, respectively, in the thicker films.
The dependences of $I_{\mathrm{ph}}, R_{\mathrm{s}}$, and $R_{\mathrm{sh}}$ on thickness also help us to better interpret the thickness-dependent PV properties presented in Table 1 . First, the output $I_{\mathrm{SC}}$ decreases with thickness, which is well attributed to the decrease in $I_{\mathrm{ph}}$ and the increase in $R_{\mathrm{s}}$. In addition, $V_{\mathrm{OC}}$ remains almost unchanged as thickness increases, probably because the reduction in $I_{\mathrm{ph}}$ is offset by the increase in $R_{\mathrm{sh}}$. Furthermore, the value of FF roughly decreases with thickness probably because the increasing rate of $R_{\mathrm{s}}$ 

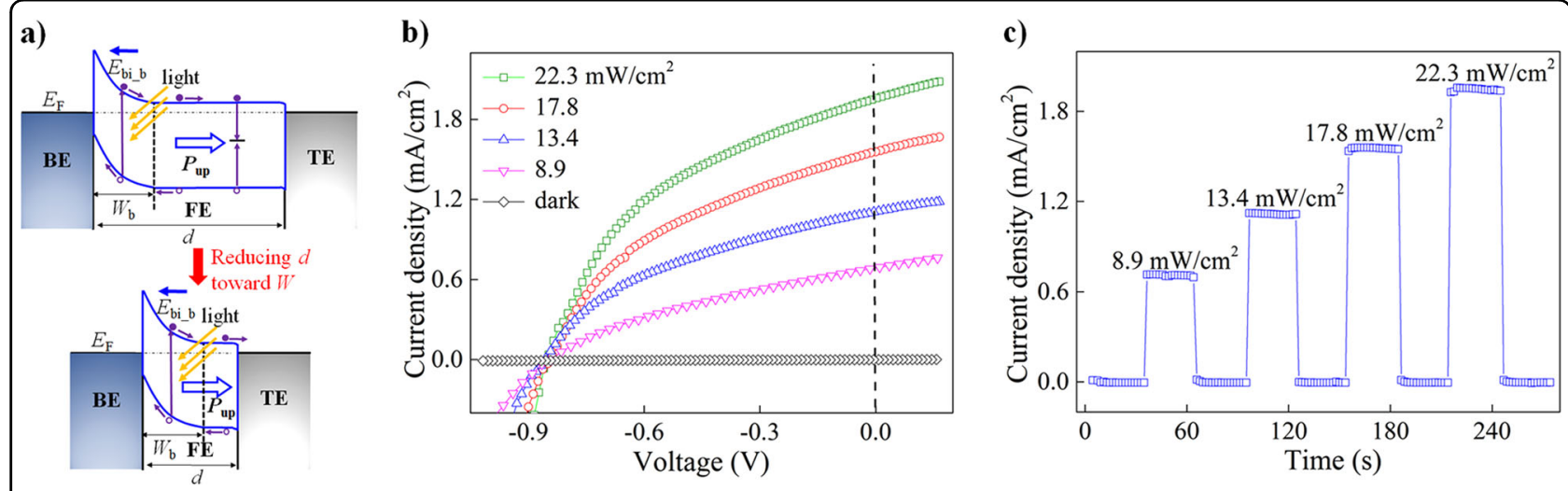

Fig. 8 Photovoltaic performance of ultrathin PZT films. a Schematics illustrating the effects of reducing the film thickness toward the depletion width on the PV process, where the recombination in the bulk region can be reduced. Here, only the bulk trap-assisted recombination is schematically drawn, but other types of recombination may also exist. b llluminated $J-V$ characteristics of the Pt/PZT (12 nm)/SRO structure at different light intensities. c Time-resolved photocurrent responses of the Pt/PZT (12 nm)/SRO structure under cyclic ON/OFF illuminations with different light intensities

is larger than that of $R_{\mathrm{sh}}$. Finally, because $\mathrm{PCE}=J_{\mathrm{SC}} \times$ $V_{\mathrm{OC}} \times \mathrm{FF} / P_{\text {light }}$, the PCE thus decreases with thickness.

\section{High PCE in the 12-nm ultrathin film}

The above thickness-dependent PV characteristics together with the fittings have revealed that the Schottky barrier effect is the dominant PV mechanism. For the Schottky barrier-driven PV effect, as proposed in the Introduction section, reducing the thickness to the depletion width may realize high PCEs mainly because the recombination outside the depletion region can be greatly suppressed (Fig. 8a). Moreover, the reduced recombination can overcompensate for the loss in light absorption, resulting in an enhancement in $I_{\mathrm{ph}}$, as evidenced by the increase in $I_{\mathrm{ph}}$ with decreasing thickness (Table 1). In addition, reducing thickness also lowers $R_{\mathrm{s}}$ because the bulk resistance is lowered (Table 1). The high $I_{\mathrm{ph}}$ together with the low $R_{\mathrm{s}}$ therefore results in a high output $I_{\mathrm{SC}}$. However, reducing thickness also lowers $R_{\mathrm{sh}}$, which would deteriorate $V_{\mathrm{OC}}$. This problem may be addressed by using small-area top electrodes to avoid the shunt paths. Therefore, a high PCE could be achieved in a ferroelectric ultrathin film with thickness close to the depletion width covered by small-area top electrodes.

To demonstrate the high PCE experimentally, we fabricated 12-nm PZT films covered by Pt top electrodes with $\sim 5 \mu \mathrm{m}$ in diameter (Fig. S2 in Supplementary Information). Notably, the thickness of $12 \mathrm{~nm}$ is close to the values of $W_{\mathrm{b}}$ and $W_{\mathrm{t}}(5.4$ and $3.2 \mathrm{~nm}$, respectively). The $J-V$ characteristics at different light intensities are shown in Fig. 8b. As the light intensity increases from 8.9 to $22.3 \mathrm{~mW} / \mathrm{cm}^{2}, J_{\mathrm{SC}}$ increases correspondingly from 0.68 to $1.94 \mathrm{~mA} / \mathrm{cm}^{2}$, while the $V_{\mathrm{OC}}$ remains at $\sim-0.84 \mathrm{~V}$. Compared with the 120-nm film, the 12-nm ultrathin film exhibits a nearly 6.5 -fold enhancement in $J_{\mathrm{SC}}$ per unit light intensity, which is well attributed to the reduced recombination and lower areal series resistance. On the other hand, $V_{\mathrm{OC}}$ slightly decreases from -0.9 to $-0.84 \mathrm{~V}$ as a result of the lower areal shunt resistance. In addition, as revealed by Fig. 8c, the photoresponses of the 12-nm PZT film are stable and reproducible.

The PCE of the 12-nm PZT film was calculated to be $2.49 \%$ (under the $22.3-\mathrm{mW} / \mathrm{cm}^{2} \mathrm{UV}$ illumination), which is one order of magnitude higher than the highest PCE, previously reported for PZT films $(\sim 0.28 \%)^{22}$ under UV illumination. To confirm that the PCE enhancement was truly a result of the thickness reduction, four additional PZT films $(4,7,30$, and $60 \mathrm{~nm})$ were prepared, and their PV properties were also characterized (see the results in Fig. S3 in Supplementary Information).

Note that according to the signs of $V_{\mathrm{OC}}$ and $J_{\mathrm{SC}}$, we deduced that the PV effect in the Pt/PZT $(12 \mathrm{~nm}) / \mathrm{SRO}$ structure originates from the PZT/SRO barrier (see Fig. 8a). This phenomenon is because the initial polarization of the 12-nm PZT film is oriented upward, leading to band alignment with an enhanced PZT/SRO barrier and a flattened Pt/PZT barrier. Thus, an efficient PV effect can occur in the PZT/SRO barrier. Unfortunately, no switchable PV behavior is observed in the Pt/PZT $(12 \mathrm{~nm}) / \mathrm{SRO}$ structure because polarization can hardly be switched downward with the top electrode capping (see Fig. S4 in Supplementary Information). This difficulty is probably caused by leakage and insufficient screening.

Nevertheless, the PV effect in the bare 12-nm PZT film is indeed switchable, as demonstrated by C-AFM and SKPM. Figure 9a presents the short-circuit current map measured by C-AFM under UV illumination, which reveals a contrast between the $+6 \mathrm{~V}$-written region $(2 \times$ 

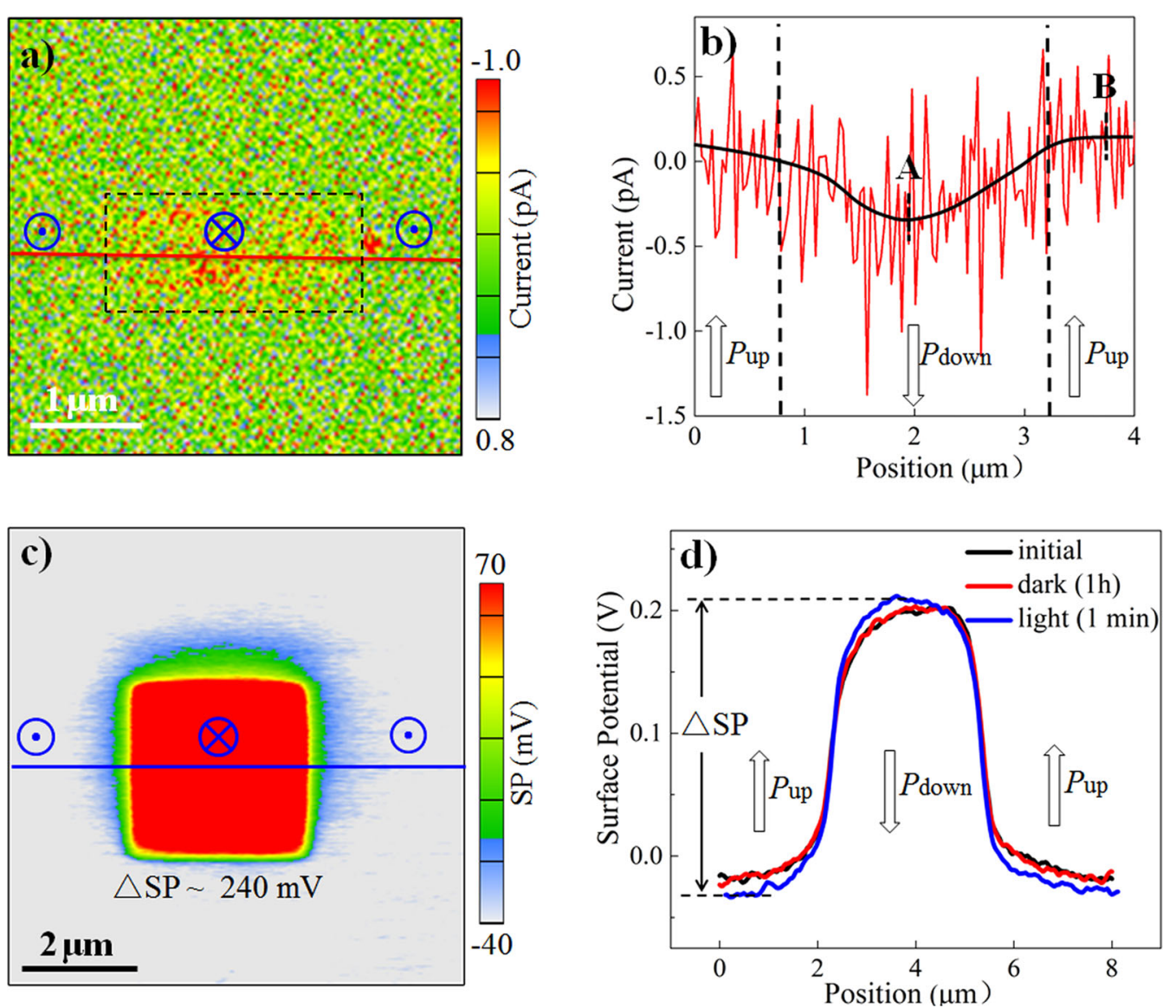

Fig. 9 C-AFM and SKPM mappings on the bare 12-nm PZT film. a Short-circuit current map generated by C-AFM under UV illumination. b Current profile along the section line indicated in panel a. The black solid line is drawn for better visualization. c Surface potential map generated by SKPM after UV illumination for 1 minute. $\mathbf{d}$ Surface potential profiles along the section lines indicated in panel a and Fig. S6 in Supplementary Information. In panels a and c, the middle $2 \times 1$ and $3 \times 3 \mu \mathrm{m}^{2}$ regions, respectively, were written with $+6 \mathrm{~V}$ beforehand

$\left.1 \mu \mathrm{m}^{2}\right)$ and the as-grown region. The current profile along the section line (Fig. 9b) further shows that the $+6 \mathrm{~V}$ written region mainly exhibits negative $I_{\mathrm{SC}}(\sim-0.31 \mathrm{pA}$ at position A), while the as-grown region mainly exhibits positive $I_{\mathrm{SC}}(\sim 0.18 \mathrm{pA}$ at position $\mathrm{B})$. Because the asgrown and $+6 \mathrm{~V}$-written regions correspond to the $P_{\text {up }}$ and $P_{\text {down }}$ states, respectively (see Fig. S5 in Supplementary Information), the C-AFM results thus indicate that the photocurrent in the $P_{\text {up }}$ state is flowing from top to the bottom, but its direction is flipped in the $P_{\text {down }}$ state (note: the average current at zero bias in the dark is set to zero, and almost no contrast is found in the dark current map, as shown in Fig. S5 in Supplementary Information; and the current direction is defined to be positive when it flows from top to the bottom).

In addition, SKPM was conducted to monitor the variation in surface potential arising from the PV effect in the bare $12-\mathrm{nm}$ PZT film. A $3 \times 3-\mu \mathrm{m}^{2}$ region was written with $+6 \mathrm{~V}$ and subsequently scanned with a grounded tip to remove mobile charges. Then, SKPM was performed to generate a surface potential map. The $+6 \mathrm{~V}$-written region exhibits higher surface potential than that of the as-grown region, and the surface potential difference $(\Delta S P)$ is $\sim 210 \mathrm{mV}$ (see Fig. S6 in Supplementary Information). This $\Delta S P$ value remains almost unchanged after the film is left in the dark for $1 \mathrm{~h}$ (see Fig. S6 in Supplementary Information), but increases to $\sim 240 \mathrm{mV}$ after UV illumination for $1 \mathrm{~min}$ (see Fig. 9c, d), suggesting the occurrence of the PV effect under UV illumination. More specifically, the photoexcited holes (electrons) are driven to the film surface and trapped there in the $+6 \mathrm{~V}$-written (asgrown) region, thus raising (lowering) the surface potential. That is, the negative (positive) photocurrent is produced in the $P_{\text {down }}\left(P_{\text {up }}\right)$ state under UV illumination, consistent with the C-AFM results shown above. Therefore, the PV effect is confirmed to be indeed switchable in the bare 12-nm PZT film. Further research is still needed to address the nonswitchability of the PV effect in the Pt electrode-capped 12-nm PZT film. 


\section{Discussion on further improvement of PCE}

To further improve the PCE requires a more thorough examination of the PV mechanism, i.e., the Schottky barrier model. From Eq. (2) and the fitting results (Table 1), one can infer that $I_{\mathrm{ph}}$ is the key to the PCE. Assuming that the recombination is neglected, the $I_{\mathrm{ph}}$ generated by a Schottky barrier is given by ${ }^{31}$

$$
\begin{aligned}
& I_{t}=A \eta n_{p h} \theta_{t}\left[1-\exp \left(-\alpha W_{t}\right)\right] q, \\
& I_{b}=A \eta n_{p h} \theta_{t} \exp \left[-\alpha\left(d-W_{b}\right)\right]\left[1-\exp \left(-\alpha W_{b}\right)\right] q,
\end{aligned}
$$

where $\eta$ is the internal quantum efficiency (assuming that $\eta=1), n_{\mathrm{ph}}$ is the incident photon flux, $\theta_{\mathrm{t}}$ is the transmittance of the top electrode, and $\alpha$ is the absorption coefficient of the ferroelectric film. Equation (3) applies to the case of $P_{\text {down }}$, where the photocurrent $I_{\mathrm{t}}$ is generated by the top barrier, whereas Eq. (4) applies to the reverse case.

For the ferroelectric ultrathin films where $d \approx W_{\mathrm{b}}$, the $\exp \left[\alpha\left(d-W_{\mathrm{b}}\right)\right]$ term in Eq. (4) may be neglected. Hence, both Eqs. (3) and (4) indicate that $I_{\mathrm{ph}}$ depends strongly on the product of $\alpha$ and $W$. To enhance $I_{\mathrm{ph}}$ and, consequently, the PCE, both the $\alpha$ and $W$ of the PZT films need to be optimized. Our PZT films have a small $\alpha$ value $\left(\sim 1.15 \times 10^{4} \mathrm{~cm}^{-1}\right.$ at $\left.365 \mathrm{~nm}\right)$ because of their wide bandgap $(\sim 3.63 \mathrm{eV})$, as measured by UV-vis spectroscopy (Fig. S7 in Supplementary Information). Narrowing the bandgap and introducing gap states by methods such as forming solid solutions ${ }^{6,7}$ and doping ${ }^{60,61}$ may effectively enhance $\alpha$. On the other hand, increasing $W$ can enhance $I_{\mathrm{ph}}$ according to Eqs. (3) and (4), but $W$ cannot be too large; otherwise, the recombination in the depletion region becomes significant, making Eqs. (3) and (4) no longer applicable. An optimal value for $W$ might be the mean free path of the ferroelectric film $(\sim 20 \mathrm{~nm}$ for $\mathrm{PZT})^{39}$. In our PZT films, $W_{\mathrm{t}}$ and $W_{\mathrm{b}}$ were calculated to be $3.2 \mathrm{~nm}$ and $5.4 \mathrm{~nm}$, respectively. Therefore, the depletion widths still need to be increased according to Eq. (1). Several approaches may be adopted: (i) reducing $N_{\text {eff }}$ by tuning the growth conditions of the PZT films (e.g., tuning the oxygen pressure during the PLD can modify the concentration of oxygen vacancies and, consequently, the $N_{\text {eff }}$ ); (ii) enhancing polarization by improving the qualities of the PZT films ${ }^{46}$ to increase $V_{\text {bi }}$ [see Eq. (S5) in Supplementary Information]; and (iii) using high-workfunction metals as electrodes and, more interestingly, optimizing the terminations of electrode/ferroelectric interfaces ${ }^{62,63}$ to increase $V_{\mathrm{bi}}$.

Note that by substituting the above $\alpha$ and $W$ values of the PZT films and the $\theta_{\mathrm{t}}$ value of the Pt electrode ( 40\%) into Eqs. (3) and (4), one can obtain the values of $I_{\mathrm{t}}$ and $I_{\mathrm{b}}$, which are $2.3 \times 10^{-8}$ and $3.4 \times 10^{-8} \mathrm{~A}$, respectively. These values are approximately one order of magnitude smaller than the $I_{\mathrm{ph}}$ values extracted from the experimental $I-V$ data (see Fig. 7a, b). The discrepancies may be mainly due to the following two issues: (i) there are errors in measuring the $\alpha$ value, although it seems to fall in the range of $10^{4}-10^{5} \mathrm{~cm}^{-1}$ reported for the PZT films ${ }^{64-66}$; and (ii) the actual area that absorbs the photons and produces the photocurrent is larger than the electrode area. Nevertheless, our strategies to achieve high PCEs suggested by the Schottky barrier model [Eqs. (2-4)], including increasing $\alpha$, optimizing $W$, and reducing the thickness to $W$, remain unaffected and may shed light on further research in the field of ferroelectric PVs. Our strategies may also synergize with other approaches, such as utilizing the flexoelectric effect arising from the nanoscale strain gradient ${ }^{67}$ and domain engineering ${ }^{68}$, to further enhance the PCEs.

\section{Conclusion}

In summary, we have demonstrated that a PCE as high as $2.49 \%$ (under $365-\mathrm{nm}$ UV illumination) could be achieved in 12-nm PZT ultrathin films. Our strategy to realize such a high PCE consists of reducing the film thickness to be close to the depletion width, which can simultaneously suppress recombination and lower the series resistance. Our strategy is based on the fact that the PV effect originates from the Schottky barriers, which is confirmed by observations of the weak dependence of $V_{\mathrm{OC}}$ on thickness and the increase in $I_{\mathrm{SC}}$ with decreasing thickness, as well as the good fits of illuminated $I-V$ characteristics to the Schottky barrier model. To implement our strategy, understanding the Schottky barrier parameters (particularly the depletion widths) is key. We therefore conducted a combination of $P-V, C-V$, and dark $I-V$ measurements on the $\mathrm{Pt} / \mathrm{PZT} / \mathrm{SRO}$ structures with different PZT thicknesses, gaining detailed information on the Schottky barriers. The present study provides insightful guidance on how to design and tailor ferroelectric films to achieve high PCEs within the framework of the Schottky barrier-driven PV effect. Our study also demonstrates the great potential of ferroelectrics for use in ultrathin-film PV devices, which may benefit the development of high-efficiency, low-cost, and low-weight solar cells.

\footnotetext{
Acknowledgements

Z.F. would like to thank H.Fan from SUSTech for technical support of EBL. This work is supported by the National Key Research Program of China (Nos. 2016YFA0201002 and 2016YFA0300101), State Key Program for Basic Research of China (No. 2015CB921202), National Natural Science Foundation of China (Nos. 51602110, 11674108, 51272078, 51431006, and 51561135014), Guangdong Innovative Research Team Program (No. 2013C102), Science and Technology Project of Guangdong Province (Nos. 2016B090918083, 2017B030301007, and 2015B090927006), Natural Science Foundation of Guangdong Province (No. 2016A030308019), and Science and Technology Project of Shenzhen Municipal Science and Technology Innovation Committee (GQYCZZ20150721150406). X.G., X.L., and Z.F. acknowledge the Project for
} 
Guangdong Province Universities and Colleges Pearl River Scholar Funded Scheme 2014, 2016, and 2018, respectively. Z.F. and G.Z. acknowledge the support from Guangdong Provincial Engineering Technology Research Center for Transparent Conductive Materials, MOE International Laboratory for Optical Information Technologies, and the 111 Project. Z.T. acknowledges the support from the Innovation Project of the Graduate School of South China Normal University.

\section{Author details}

Institute for Advanced Materials, South China Academy of Advanced Optoelectronics, South China Normal University, Guangzhou 510006, China. ${ }^{2}$ Guangdong Provincial Key Laboratory of Optical Information Materials and Technology, South China Academy of Advanced Optoelectronics, South China Normal University, Guangzhou 510006, China. ${ }^{3}$ Department of Industrial Systems Engineering and Management, National University of Singapore, Singapore 117576, Singapore. ${ }^{4}$ National Center for International Research on Green Optoelectronics, South China Normal University, Guangzhou 510006 China. ${ }^{5}$ Laboratory of Solid State Microstructures and Innovation Center of Advanced Microstructures, Nanjing University, Nanjing 210093, China

\section{Conflict of interest}

The authors declare that they have no conflict of interest.

\section{Publisher's note}

Springer Nature remains neutral with regard to jurisdictional claims in published maps and institutional affiliations.

Supplementary information is available for this paper at https://doi.org/ 10.1038/s41427-019-0120-3.

Received: 15 December 2018 Revised: 1 February 2019 Accepted: 6 February 2019.

Published online: 26 April 2019

\section{References}

1. Lopez-Varo, P. et al. Physical aspects of ferroelectric semiconductors for photovoltaic solar energy conversion. Phys. Reports. 653, 1-40 (2016).

2. Paillard, C. et al. Photovoltaics with ferroelectrics: current status and beyond. Adv. Mater. 28, 5153-5168 (2016).

3. Fan., Z., Sun, K. \& Wang, J. Perovskites for photovoltaics: a combined review of organic-inorganic halide perovskites and ferroelectric oxide perovskites. J. Mater. Chem. A 3, 18809-18828 (2015).

4. Yuan, Y., Xiao, Z., Yang, B. \& Huang, J. Arising applications of ferroelectric materials in photovoltaic devices. J. Mater. Chem. A 2, 6027-6041 (2014).

5. Li, J. et al. Touching is believing: interrogating halide perovskite solar cells at the nanoscale via scanning probe microscopy. npj Quantum Materials 2, 56 (2017).

6. Nechache, R. et al. Bandgap tuning of multiferroic oxide solar cells. Nat. Photonics. 9, 61-67 (2014).

7. Grinberg, I. et al. Perovskite oxides for visible-light-absorbing ferroelectric and photovoltaic materials. Nature 503, 509-512 (2013).

8. Huang, B. et al. Ferroic domains regulate photocurrent in single-crystalline $\mathrm{CH}_{3} \mathrm{NH}_{3} \mathrm{Pbl}_{3}$ films self-grown on $\mathrm{FTO} / \mathrm{TiO}_{2}$ substrate. npj Quantum Materials 3 , 30 (2018).

9. Li, J.-K. et al. Self-driven visible-blind photodetector based on ferroelectric perovskite oxides. Appl. Phys. Lett. 110, 142901 (2017).

10. Gan, B. K., Yao, K., Lai, S. C., Chen, Y. F. \& Goh, P. C. An ultraviolet (UV) detector using a ferroelectric thin film with in-plane polarization. IEEE Electron Device Lett 29, 1215-1217 (2008).

11. Guo, R. et al. Non-volatile memory based on the ferroelectric photovoltaic effect. Nat. Commun. 4, 1990 (2013).

12. Thakoor, S. High speed, nondestructive readout from thin-film ferroelectric memory. Appl. Phys. Lett. 60, 3319 (1992).

13. Ji, W., Yao, K. \& Liang, Y. C. Bulk photovoltaic effect at visible wavelength in epitaxial ferroelectric $\mathrm{BiFeO}_{3}$ thin films. Adv. Mater. 22, 1763-1766 (2010).

14. Choi, T., Lee, S., Choi, Y. J., Kiryukin, V. \& Cheong, S.-W. Switchable ferroelectric diode and photovoltaic effect in $\mathrm{BiFeO}_{3}$. Science 324, 63-66 (2009).
15. Yang, S. Y. et al. Above-bandgap voltages from ferroelectric photovoltaic devices. Nat. Nanotechnol. 5, 143-147 (2010).

16. Bhatnagar, A., Chaudhuri, A. R., Kim, Y. H., Hesse, D. \& Alexe, M. Role of domain walls in the abnormal photovoltaic effect in $\mathrm{BiFeO}_{3}$. Nat. Commun. 4, 2835 (2013).

17. Spanier, J. E. et al. Power conversion efficiency exceeding the ShockleyQueisser limit in a ferroelectric insulator. Nat. Photonics. 10, 611-616 (2016).

18. Nechache, R., Huang, W., Li, S. \& Rosei, F. Photovoltaic properties of $\mathrm{Bi}_{2} \mathrm{FeCrO}_{6}$ films epitaxially grown on (100)-oriented silicon substrates. Nanoscale. 8, 3237-3243 (2016).

19. Zheng, F. et al. Above $1 \%$ efficiency of a ferroelectric solar cell based on the $\mathrm{Pb}$ $(\mathrm{Zr}, \mathrm{Ti}) \mathrm{O}_{3}$ film. J. Mater. Chem. A 2, 1363-1368 (2014).

20. Cao, D. et al. High-efficiency ferroelectric-film solar cells with an n-type $\mathrm{Cu}_{2} \mathrm{O}$ cathode buffer layer. Nano. Lett. 12, 2803-2809 (2012).

21. Fan, Z., Yao, K. \& Wang, J. Photovoltaic effect in an indium-tin-oxide/ZnO/ $\mathrm{BiFeO}_{3} /$ Pt heterostructure. Appl. Phys. Lett. 105, 162903 (2014).

22. Qin, M., Yao, K. \& Liang, Y. C. High efficient photovoltaics in nanoscaled ferroelectric thin films. Appl. Phys. Lett. 93, 122904 (2008).

23. Fan, $\mathrm{H}$. et al. Large electroresistance and tunable photovoltaic properties of ferroelectric nanoscale capacitors based on ultrathin super-tetragonal $\mathrm{BiFeO}_{3}$ films. J. Mater. Chem. C 5, 3323-3329 (2017).

24. Zenkevich, A. et al. Giant bulk photovoltaic effect in thin ferroelectric BaTio3 films. Phys. Rev. B 90, 161409 (R) (2014).

25. Fridkin, V. M. \& Popov, BN Anomalous photovoltaic effect in ferroelectrics. Sov. Phys. Usp. 21, 981-991 (1978).

26. Gu, Z O et al. Mesoscopic free path of nonthermalized photogenerated carriers in a ferroelectric insulator. Phys. Rev. Lett. 118, 096601 (2017).

27. Ji, W., Yao, K. \& Liang, Y. C. Evidence of bulk photovoltaic effect and large tensor coefficient in ferroelectric $\mathrm{BiFeO}_{3}$ thin films. Phys. Rev. B 84, 094115 (2011).

28. Qin, M., Yao, K. \& Liang, Y. C. Photovoltaic mechanisms in ferroelectric thin films with the effects of the electrodes and interfaces. Appl. Phys. Lett. 95 022912 (2008).

29. Qin, M., Yao, K. \& Liang, Y. C. Photovoltaic characteristics in polycrystalline and epitaxial $\left(\mathrm{Pb}_{0.97} \mathrm{La}_{0.03}\right)\left(\mathrm{Zr}_{0.52} \mathrm{Ti}_{0.48}\right) \mathrm{O}_{3}$ ferroelectric thin films sandwiched between different top and bottom electrodes. J. Appl. Phys. 105, 061624 (2009).

30. Yang, Y. S. et al. Schottky barrier effects in the photocurrent of sol-gel derived lead zirconate titanate thin film capacitors. Appl. Phys. Lett. 76, 774 (2000).

31. Pintilie, L., Dragoi, C. \& Pintilie, I. Interface controlled photovoltaic effect in epitaxial $\mathrm{Pb}(\mathrm{Zr}, \mathrm{Ti}) \mathrm{O}_{3}$ films with tetragonal structure. J. Appl. Phys. 110, 044105 (2011).

32. Fang, L. et al. Switchable photovoltaic response from polarization modulated interfaces in $\mathrm{BiFeO}_{3}$ thin films. Appl. Phys. Lett. 104, 142903 (2014).

33. Pintilie, L., Vrejoiu, I., Rhun, G. L. \& Alexe, M. Short-circuit photocurrent in epitaxial lead zirconate-titanate thin films. J. Appl. Phys. 101, 064109 (2007).

34. Yang, S. Y. et al. Photovoltaic effects in $\mathrm{BiFeO}_{3}$. Appl. Phys. Lett. 95, 062909 (2009).

35. Lee, D. et al. Polarity control of carrier injection at ferroelectric/metal interfaces for electrically switchable diode and photovoltaic effects. Phys. Rev. B 84, 125305 (2011).

36. Seidel, J. et al. Efficient photovoltaic current generation at ferroelectric domain walls. Phys. Rev. Lett. 107, 126805 (2011)

37. Guo, R., You, L., Chen, L., Wu, D. \& Wang, J. Photovoltaic property of BiFeO thin films with 109 domains. Appl. Phys. Lett. 99, 122902 (2011).

38. Zheng, F., Xu, J., Fang, L., Shen, M. \& Wu, X. Separation of the Schottky barrier and polarization effects on the photocurrent of $\mathrm{Pt}$ sandwiched $\mathrm{Pb}\left(\mathrm{Zr}_{0.20} \mathrm{Ti}_{0.80}\right)$ $\mathrm{O}_{3}$ films. Appl. Phys. Lett. 93, 172101 (2008).

39. Pintilie, L., Vrejoiu, I., Hesse, D., LeRhun, G. \& Alexe, M. Ferroelectric polarizationleakage current relation in high quality epitaxial $\mathrm{Pb}(\mathrm{Zr}, \mathrm{Ti}) \mathrm{O}_{3}$ films. Phys. Rev. B 75, 104103 (2007)

40. Walker, D., Thomas, P. A. \& Collins, S. P. A comprehensive investigation of the structural properties of ferroelectric $\mathrm{PbZr}_{0.2} \mathrm{Ti}_{0.8} \mathrm{O}_{3}$ thin films grown by $\mathrm{PLD}$. Phys. Status Solidi A 206, 1799-1803 (2009).

41. Pintilie, $\mathrm{L}$. et al. Polarization induced self-doping in epitaxial $\mathrm{Pb}\left(\mathrm{Zr}_{0.20} \mathrm{Ti}_{080}\right) \mathrm{O}_{3}$ thin films. Sci. Rep. 5, 14974 (2015)

42. Lee, H. N. et al. Suppressed dependence of polarization on epitaxial strain in highly polar ferroelectrics. Phys. Rev. Lett. 98, 217602 (2007).

43. Železný, $\vee$. et al. The variation of $\mathrm{PbTiO}_{3}$ bandgap at ferroelectric phase transition. J. Phys. Condens. Matter. 28, 025501 (2016) 
44. Khan, A. I., Marti, X., Serrao, C., Ramesh, R. \& Salahuddin, S. Voltage-controlled ferroelastic switching in $\mathrm{Pb}\left(\mathrm{Zr}_{02} \mathrm{Ti}_{08}\right) \mathrm{O}_{3}$ thin films. Nano. Lett. 15, 2229-2234 (2015).

45. Rhun, G. L. et al. Increased ferroelastic domain mobility in ferroelectric thin films and its use in nano-patterned capacitors. Nanotech. 17, 3154 (2006).

46. Vrejoiu, B. I. et al. Intrinsic ferroelectric properties of strained tetragonal $\mathrm{PbZr}_{0.2} \mathrm{Ti}_{0.8} \mathrm{O}_{3}$ obtained on layer-by-layer Grown, defect-free single-crystalline films. Adv. Mater. 18, 1657-1661 (2006).

47. Tagantsev, A. K., Pawlaczyk, C., Brooks, K. \& Setter, N. Built-in electric field assisted nucleation and coercive fields in ferroelectric thin films. Integrated Ferroelectrics 4, 1-12 (1994).

48. Tagantsev, A. K. \& Gerra, G. Interface-induced phenomena in polarization response of ferroelectric thin films. J. Appl. Phys. 100, 051607 (2006).

49. Pintilie, L. \& Alexe, M. Metal-ferroelectric-metal heterostructures with Schottky contacts. I. Influence of the ferroelectric properties. J. Appl. Phys. 98, 124103 (2005).

50. Pintilie, L. et al. Metal-ferroelectric-metal structures with Schottky contacts. II. Analysis of the experimental current-voltage and capacitance-voltage characteristics of $\mathrm{Pb}(\mathrm{Zr}, \mathrm{Ti}) \mathrm{O}_{3}$ thin films. J. Appl. Phys. 98, 124104 (2005).

51. Li, P. et al. An unusual mechanism for negative differential resistance in ferroelectric nanocapacitors: polarization switching-induced charge injection followed by charge trapping. ACS Appl. Mater. Interfaces 9, 27120-27126 (2017).

52. Zubko, $\mathrm{P}_{.}$Jung, D. J. \& Scott, J. F. Electrical characterization of $\mathrm{PbZr}_{04} \mathrm{Ti}_{06} \mathrm{O}_{3}$ capacitors. J. Appl. Phys. 100, 114113 (2006).

53. Scott, J. F., Watanabe, K., Hartmann, A. J. \& Lamb, R. N. Device models for PZT/ PT, BST/PT, SBT/PT, and SBT/BI ferroelectric memories. Ferroelectrics 225, 83-90 (1999).

54. Jiang, A. Q. et al. A resistive memory in semiconducting $\mathrm{BiFeO}_{3}$ thin-film capacitors. Adv. Mater. 23, 1277-1281 (2011)

55. Fang, L. et al. Switchable photovoltaic response from polarization modulated interfaces in $\mathrm{BiFeO}_{3}$ thin films. Appl. Phys. Lett. 104, 142903 (2014).
56. Fan, Z. et al. Enhanced photovoltaic effects and switchable conduction behavior in $\mathrm{BiFe}_{06} \mathrm{SC}_{04} \mathrm{O}_{3}$ thin films. Acta Mater. 88, 83-90 (2015).

57. Jain, A. \& Kapoor, A. Exact analytical solutions of the parameters of rea solar cells using Lambert W-function. Sol. Energy Mater. Sol. Cells 81, 269-277 (2004).

58. Ortiz-Conde, A., Sanchez, F. J. G. \& Muci, J. New method to extract the model parameters of solar cells from the explicit analytic solutions of their illuminated I-V characteristics. Sol. Energy Mater. Sol. Cells 90, 352-361 (2006)

59. Zhang, C., Zhang, J., Hao, Y., Lin, Z. \& Zhu, C. A simple and efficient solar cell parameter extraction method from a single current-voltage curve. J. Appl. Phys. 110, 064504 (2011)

60. Choi, W. S. et al. Wide bandgap tunability in complex transition metal oxides by site-specific substitution. Nat. Commun. 3, 689 (2012).

61. Matsuo, H., Noguchi, Y. \& Miyayama, M. Gap-state engineering of visible-lightactive ferroelectrics for photovoltaic applications. Nat. Commun. 8, 207 (2017).

62. Susaki, T., Makishima, A. \& Hosono, H. Work function engineering via $\mathrm{LaAlO}_{3} /$ $\mathrm{SrTiO}_{3}$ polar interfaces. Phys. Rev. B 84, 115456 (2011).

63. Guo, R. et al. Control of synaptic plasticity learning of ferroelectric tunnel memristor by nanoscale interface engineering. ACS Appl. Mater. Interfaces $\mathbf{1 0}$ 12862 (2018).

64. Peng, C. H., Chang, J.-F. \& Desu, S. B. Optical properties of PZT, PLZT, and PNZT thin films. Mat. Res. Soc. Sym. Pro. 243, 21 (1992).

65. Jiang, Y. P., Tang, X. G., Liu, Q. X., Li, Q. \& Ding, A. L. Optical properties of Pb $\left(\mathrm{Zr}_{0.53} \mathrm{Ti}_{0.47}\right) \mathrm{O}_{3}$ thin films on Pt-coated Si substrates measured by spectroscopic ellipsometry in the UV-vis-NIR region. Mater. Sci. Eng. B 137, 304-309 (2007).

66. Lee, $\mathrm{H}$. et al. Dielectric functions and electronic band structure of lead zirconate titanate thin films. J. Appl. Phys. 98, 094108 (2005).

67. Chu, K. et al. Enhancement of the anisotropic photocurrent in ferroelectric oxides by strain gradients. Nature Nanotech. 10, 972 (2015).

68. Sung, J. $\mathrm{H}$. et al. Single ferroelectric-domain photovoltaic switch based on lateral $\mathrm{BiFeO}_{3}$ cells. NPG Asia Mater. 5, e38 (2013). 